\title{
Analyses of 94 consecutive spinal cord injury patients using ASIA definition and modified Frankel score classification
}

\author{
M Capaul MD,${ }^{1} \mathrm{H}$ Zollinger MD,${ }^{1} \mathrm{~N}$ Satz MD,${ }^{1} \mathrm{~V}$ Dietz MD,${ }^{1} \mathrm{D}$ Lehmann MD,${ }^{2}$ \\ B Schurch MD ${ }^{1}$ \\ ${ }^{1}$ Department of Orthopedics and Swiss Paraplegic Center, University Hospital Balgrist, \\ Zurich, Forchstrasse 340, 8008 Zurich, Switzerland; ${ }^{2}$ Department of Neurology, \\ University Hospital, Zurich, Switzerland.
}

Serial neurological examinations were analysed on 94 consecutive spinal cord injury (SCI) patients admitted for rehabilitation to the Swiss Paraplegic Center at the Clinic Balgrist Zurich, Switzerland, between 1987 and 1992. Patients' data were examined adopting ASIA and modified Frankel definitions in order to compare the two classifications in terms of consistency and prognostic value. The modified Frankel definition was subdivided into five categories (A, B, C, D and E). On admission (discharge) 43 (37) patients were classified as Frankel A, 23 (11) patients in group B, 26 (42) patients in group C, 2 (2) patients as Frankel D and 0 (2) patients in group E. A qualitative analysis of the results on the base of a maximal score of 100 points $(A=0, B=25, C=50, D=75$ and $\mathrm{E}=100$ points) suggested a mean score improvement from $21.5( \pm 22.5)$ to 29.0 $( \pm 26.3)$ or $7.5( \pm 7.1)$, regarding all 94 patients during follow up (admission/ discharge). The median improvement was one modified Frankel grade (A/B to $\mathrm{B} / \mathrm{C})$. No detailed assessments were yielded concerning motor and sensory functions. Using ASIA definition, a continuous numerical score of motor and sensory function was observed. Recovery during follow up was determined by detailed motor and sensory function. For all 94 patients (quadriplegics and tetraplegics), the average motor recovery according to the ASIA definition was 9.4 ( \pm 9.6$)$. The mean ASIA motor score improved from $52.2( \pm 17.3)$ on admission to $61.6( \pm 17.9)$ on discharge. Light touch increased by $7.0( \pm 10.3)$ from $72.7( \pm 22.3)$ to $79.7( \pm 22.7)$ and pinprick sensory function by $7.1( \pm 13.6)$ from $69.2( \pm 21.8)$ to $76.3( \pm 22.2)$. Change in status was progressively unidirectional using both definitions. Comparing the ASIA guidelines with modified Frankel classification there was an unambiguous benefit using the new definition of ASIA, as a gradual change of motor and/or sensory function was more clearly documented for all cases by ASIA. Using modified Frankel score definition, the patient's classification may be unchanged, regardless of whether the status improved or remained stable. This was not the case using ASIA definition. It was not the intention in this paper to assess and compare the treatment of acutely spinal cord injured patients by (a) nonoperative and (b) operative treatment.

Keywords: modified Frankel score definition; ASIA (American Spinal Injury Association); spinal cord injury (SCI); ASIA Motor Index Score.

\section{Introduction}

During the last 30 years several meetings have taken place worldwide in order to

Correspondence to Dr Marcel Capaul, Russenweg 18, $\mathrm{CH}-8008$ Zurich, Switzerland. elaborate an effective classification of spinal cord injury. ${ }^{1}$ Despite different methods and proposals no valid solution was agreed upon. ${ }^{2.3}$ The modified Frankel score was preferably used. Using the modified Frankel score, complete and incomplete spinal cord 
injury were subdivided into five categories (A, B, C, D and E), without yielding detailed assessments of motor and sensory function.

Recently the American Spinal Injury Association (ASIA) worked out a new classification of spinal cord injury based on the ASIA Motor Score Index. Motor function, light touch and pinprick sensory function were scored and analysed by a different points rationing scheme. ${ }^{4}$

According to the Standards for Neurological Classification published by ASIA, the term 'complete injury' is used when there is an absence of sensory and motor function in the lowest sacral segment; 'incomplete injury' means partial preservation of sensory and/or motor function below the neurological level including the lowest sacral segment. Sacral sensation includes sensation at the anal mucocutaneus junction as well as deep anal sensation. The test for motor function is the presence of voluntary contraction of the external anal sphincter upon digital examination. ${ }^{4}$

In the present paper, the two classifications (ASIA/Frankel) were compared and the patients' data were separately analysed.

\section{Methods and subjects}

Serial examinations for motor and sensory function were performed on 94 consecutive spinal cord vertebral injury patients admitted for treatment and rehabilitation to the University Hospital Department of Orthopedics and Swiss Paraplegic Center Balgrist, Zurich between 1987 and 1992.

For this study the data on admission and at discharge were used in order to compare the definitions of the modified Frankel score with ASIA classification in terms of consistency and prognostic ability. ${ }^{5}$ The ASIA Motor Index Score uses standard manual muscle testing on a six grade scale (absent $=0 ; \quad$ trace $=1$, visible or palpable contraction; poor $=2$, active movement through range of motion with gravidity eliminated; fair $=3$, active movement through range of motion against gravidity; good $=4$, active movement through range of motion against resistance; normal $=5$; and $\mathrm{NT}=$ not testable). The key muscles/ functions included in the ASIA Motor Index Score are: elbow flexors, wrist extensors, elbow extensors, finger flexors, hand intrinsics, hip flexors, knee extensors, ankle dorsiflexors, long toe extensors and ankle plantar flexors, whereas a total motor score of 100 is possible. The sensory dermatome chart recommended by ASIA was used. Light touch and pinprick sensations were tested for each sensory dermatome and graded on a three point scale (absent $=0$; impaired $=1$; and normal $=2$ ). NT was used for not testable. Quantitatively a maximum light touch score of 112, pinprick score of 112 is possible. Bladder, bowel and sexual dysfunctions were separately analysed.

For the modified Frankel score ${ }^{6}{ }^{2}$ a five scale subdivision was used: $\mathrm{A}=$ complete motor and sensory function disorder; $\mathrm{B}=$ motor complete, sensory incomplete function disorder; $\mathrm{C}=$ motor and sensory incomplete function disorder; $\mathrm{D}=$ useful motor function with or without auxiliary means; $E=$ no motor or sensory function disorder.

In order to get a qualitative analysis, a 100 point scale was used $(\mathrm{A}=0, \mathrm{~B}=25$, $\mathrm{C}=50, \mathrm{D}=75$ and $\mathrm{E}=100$ points). By this means, a patient with complete motor and sensory function disorder (Frankel A) was classified with no points, whereas a patient with, for instance, a complete motor and sensory function recovery (Frankel E) was classified with the maximal score of 100 points. Patients were classified on both scores (ASIA and Frankel) according to their initial neurological level of injury on admission and their final condition at discharge.

The ASIA Motor Index increase between initial and final exams was used as an indicator of motor recovery. The increase between initial and final light touch and pinprick scores was used to measure sensory recovery.

\section{Results}

The mean age of all 94 patients on admission was 37.8 years. Of this population, 21 $(22 \%)$ of the patients were women and 73 $(78 \%)$ men. In accordance with the internal 
guidelines for spinal cord injury, 28 patients $(30 \%)$ were treated conservatively, while 43 patients $(46 \%)$ were operated within $24 \mathrm{~h}$ (early decompression) and 23 of the patients $(24 \%)$ underwent surgical treatment later, after more than $24 \mathrm{~h}$ (late decompression). Without exception, all 94 patients were hospitalized within $24 \mathrm{~h}$ after spinal cord vertebral injury. All patients were directly assigned to our clinic, either by ambulance or aircraft.

\section{Type of vertebral fracture}

In 26 cases there was a dislocation of the vertebral body $(25.5 \%)$; in 24 cases $(27.5 \%)$ a dislocation-fracture; in 22 cases $(23 \%)$ a compression-fracture; in 14 cases $(15 \%)$ a burst-fracture and in eight cases $(9 \%)$ a communited-fracture.

\section{Cause of injury}

In 40 patients the cause of acute traumatic vertebral lesion with consecutive SCI was a traffic accident; in 23 patients sport accidents, in 20 patients work accidents, and in 6 patients attempted suicide were the causative mechanisms. There were five cases of vague reason.

\section{Level of spinal cord injury}

On admission 36 patients $(38 \%)$ were tetraplegic and 58 patients (62\%) paraplegic. In 12 patients $(12.7 \%)$ with a diagnosis of tetraplegia the neurological level most often affected was C6. In 10 patients $(9.4 \%)$ with the diagnosis of paraplegia the corresponding neurological injured level was L1. Thirty-two patients (94\%) having paraplegia were affected between T11 and L2. No primary spinal cord lesion was found at level C1, C8, T1 and S3-5 (Fig 1a).

\section{Level of vertebral column injury}

In the patients with tetraplegia, the most frequent affected spine level was C5 (14 patients). L1 was the location most frequently injured in the 18 patients with paraplegia. In general the thoracolumbar junction was the most affected level of vertebral column injury (25 patients) (Fig 1b).
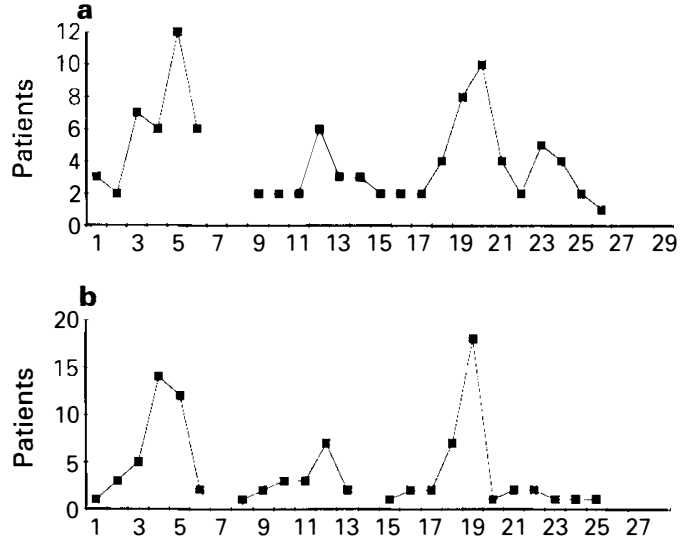

Figure 1 (a) Level of spinal cord injury (30 segments). $\mathrm{C} 1-8=1-8 ; \mathrm{T} 1-12=9-20 ; \mathrm{L} 1-5=$ $21-25 ; \mathrm{S} 1-5=26-30$. (b) Level of vertebral lesion (29 segments). $\mathrm{C} 1-7=1-7 ; \quad \mathrm{T} 1-12=$ $8-19 ; \mathrm{L} 1-5=20-24 ; \mathrm{S} 1-5=25-29$. Obviously there is a strong coincidence between the level of spinal cord injury (SCI) and the level of acute traumatic vertebral lesion. Main peaks are at C6 and L1 in patients having the diagnosis of tetraplegia and paraplegia respectively.

\section{Neurological results}

Frankel score. On admission, 43 patients were classified as Frankel A, 23 patients as Frankel B, 26 patients as Frankel C, two patients as Frankel $\mathrm{D}$ and no patient as Frankel E. At discharge, 37 patients were still classified as Frankel A (six patients changed to group B), 11 patients as Frankel B (18 patients changed to group C), 42 patients as Frankel C (two patients changed to group D), two patients as Frankel D and two patients as Frankel E (two new patients). Using the numerical scale of 100 points, there was a mean improvement from $21.5( \pm 22.5)$ to $29.0( \pm 26.3)$ or an increase of $7.5( \pm 7.1)$ considering all 94 patients. The median improvement was one modified Frankel grade (A/B to B/C).

ASIA Motor Index Score. The mean ASIA motor score on admission of all 94 patients was $52.2( \pm 17.3)$ and the last follow up mean score on discharge was 61.6 $( \pm 17.9)$ of a maximal score of 100 points. There was a mean motor point improvement of $9.4( \pm 9.6)$. For paraplegics, the average motor recovery for all 58 patients, was $4.9( \pm 6.1)$, which was numerically less 
than the average motor recovery for all 36 quadriplegics $16.9( \pm 11.2)$. Change in neurological status of complete or incomplete functional disorder was progressively unidirectional, independent of whether conservative or surgical treatment (early/late decompression) was performed. None of the $66(70 \%)$ operatively treated patients demonstrated motor point deterioration postoperatively. According to ASIA definition 27 of all 58 paraplegics showed a complete motor function disorder on admission. Two of the 27 patients had changed to incomplete status on discharge. Twentyeight out of 36 tetraplegics showed a complete motor function disorder on admission. Seven patients had changed to incomplete status and 21 patients remained in a status of complete motor function disorder on discharge.

On admission, the average score of pinprick of all 94 patients was $69.2( \pm 21.8)$ out of a maximal 112 points. At discharge 76.3 $( \pm 22.2)$ was the mean score, which was an improvement of $7.1( \pm 13.6)$. The light touch score of $72.7( \pm 22.3)$ improved to a final score of $79.7( \pm 22.7)$. There was a mean increase of $7.0( \pm 10.3)$.

Rehabilitation and walking ability. The quality of rehabilitation was divided into five categories: $1=$ only with wheelchair, $2=$ with orthoses, $3=$ with a walking stick; $4=$ without help; $5=$ no problems. At discharge 52 patients were able to move only in a wheelchair; 12 patients used orthoses; 16 patients were using a walking stick; nine patients could walk sufficiently without any help; five patients showed absolutely no walking disability (Fig 2).

Vegetative function disorder (bladder, bowel and sexual) was found in 80 patients on admission, and was still present in 67 patients on discharge.

\section{Discussion}

Immediately after spinal cord injury, a state of spinal shock develops which result in areflexia for varying time periods. A patient at this time may have no motor or sensory function below the level of the lesion, but still regain neurological functions later. ${ }^{7}$

The difference observed between the

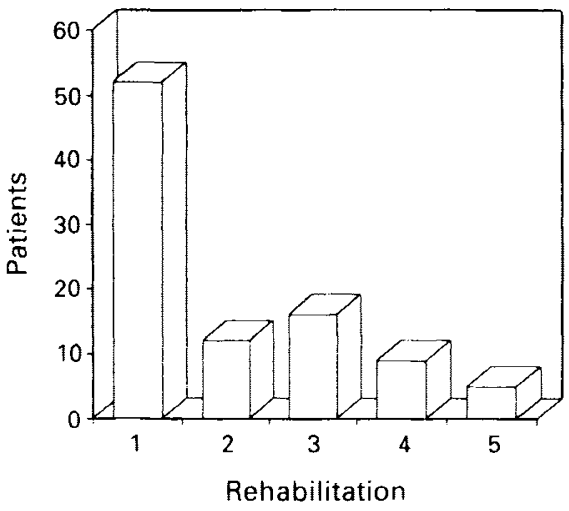

Figure 2 Rehabilitation outcome. There are five categories of rehabilitation: $1=$ invalid chair only; 2 = orthoses; 3 = walking stick; $4=$ without help; $5=$ normal.

Frankel analysis (66 patients with complete motor function disorder and with complete or incomplete sensory function disorder (43 Frankel A + 23 Frankel B)) and the ASIA motor function score (55 patients with complete motor function disorder, 27 paraplegics and 28 tetraplegics) on admission, is due to the more detailed definition of ASIA to classify motor function disorder. Patients, e.g. those with only a trace of muscle contraction in the lowest sacral segment, were determined, according to ASIA definition, as incomplete motor function disorder; meanwhile such patients were still classified in group $\mathrm{A}$ or $\mathrm{B}$ with complete motor function disorder, using the modified Frankel score.

Our study suggests that an accurate classification of recovery of patients with motor and sensory function disorder is less possible using the modified Frankel score. The mean improvement of 7.5 points, from 21.5 to 29.0 points, showed a switch from interval Frankel $\mathrm{A}-\mathrm{B}$ to $\mathrm{B}-\mathrm{C}$ on a 100 point scale, leading to a hypothetical change of function disorder of most of the patients (median) from a motor and sensory complete (Frankel A) to a motor complete and sensory incomplete function disorder (Frankel B). This did not of course correspond with the real outcome, as only six patients left group A to join group B. The principal change using Frankel score in this 
study was the improvement of 18 patients from group B to Frankel C (motor and sensory incomplete disorder).

On the contrary, a step-like graduation was not obvious using the ASIA definition, as continuous numerical scores of motor and sensory function were observed. Numerically, nine of all 94 patients (two paraplegics and seven tetraplegics) changed from complete to incomplete motor function disorder, using ASIA definition. This is contrary to the modified Frankel definition, where 18 patients changed from complete to incomplete motor function disorder (group $\mathrm{B}$ to group $\mathrm{C}$ ). However, the less detailed standardisation of modified Frankel definition using manual muscle testings revealed less accurate and higher result of motor recovery (complete to incomplete motor function disorder) than using ASIA definition.

The data referred to the ASIA classification revealed that, with increasing time after injury, a persistence of complete injury progressively diminishes the chance for motor or/and sensory recovery. Patients in this series were examined on admission and on discharge and those having a diagnosis of complete injury by ASIA definition had a relatively small amount of motor or sensory recovery during follow up in comparison to patients having a diagnosis of incomplete injury. The individual and gradual change of motor and sensory function was more clearly documented for all cases by ASIA, allowing a more useful and valid system for process analysis. Using modified Frankel score definitions, the patient's classification may be unchanged, regardless of whether the status improved or remained stable. Clear control of outcome was better assessed by ASIA definition.

The higher increase of total score points in tetraplegics compared with paraplegics, having a diagnosis of incomplete injury on admission, was obvious by counting additively the upper and lower motor function as a sum, recommended by the definition of ASIA. A complete recovery in motor and/or sensory function was only reached by patients having a minimal neurological lesion on admission after acute spinal cord injury.

As documented, ${ }^{8}$ the results of motor and sensory function tests of spinal cord injury could be rather inconsistent among examiners. As the using of ASIA guidelines showed, precise evaluation of data is the key for further elaboration of information and allows better interpretation of data taken by serial examinations.

\section{Conclusion}

Comparing the ASIA guidelines with the modified Frankel score there is an unambiguous benefit using the new classification of ASIA, provided that the conventions concerning the neurologic examination and methods of classifying patients with spinal cord injury are taken for granted.

\section{References}

1 Lucas JT, Drucker TB (1979) Motor classification of spinal cord injuries with mobility, morbidity and recovery indices. Am Surg 16: 45: 151-158.

2 Michaelis LS (1968) Discussion on classification of neurological lesions. Paraplegia 6: 46-63.

3 Michaelis LS (1969) International inquiry on neurological terminology and prognosis in paraplegia and tetraplegia. Paraplegia 7: 1-5.

4 Ditunno JF, Young W, Donovan WH, Creasey G (1994) The International Standards Booklet for Neurological and Functional Classification of Spinal Cord Injury. Paraplegia 32: 70-80.

5 Young JS, Burns PE, Bowen AM, McCutchen R (1982) Spinal cord injury statistics. Good Samaritan Medical Center, Phoenix.

6 Meinecke FW (1990) Querschnittslähmung, Bestandesaufnahme und Zukunftsaussichten. Springer Verlag, Berlin, Heidelberg.

7 Guttmann L (1963) Initial treatment of traumatic paraplegia and tetraplegia. In: Harris P, editor. Symposium on Spinal Injuries, R Coll Surg Edinburgh: 80-92.

8 Donovan WH, Wilkerson MA, Rossi D et al (1990) A test of the ASIA guidlines for classification of spinal cord injury. Neurol Rehabil 4: 39-53. 